\title{
Superoxide-Dependent Chemotactic Activity for PMNs Derived from Opsonized Zymosan- Stimulated Human Platelets
}

\author{
DOMENICO DEL PRINCIPE, ADRIANA MENICHELLI, ELENA GALLI, MAURO PERSIANI, \\ ROBERTO PERLINI, CARMELO D'ARCANGELO, LUISA BUSINCO, AND PAOLO ROSSI ${ }^{(33)}$ \\ Department of Pediatrics $I^{\circ}$, University of Rome, Rome, Italy and International Center for Interdisciplinary Studies of \\ Immunology Georgetown University School of Medicine, Washington, D.C., USA [P.R.]
}

\begin{abstract}
Summary
Previous studies have shown that platelets exhibit a $\mathrm{H}_{2} \mathrm{O}_{2}$ producing, NADH-dependent system that is activated by interaction with particulate material. Current evidence suggests that this system could be critically involved in the generation of chemotactic factor(s). In the present studies, chemotactic activity for polymorphonuclear leukocytes of supernatants derived from zymosan-stimulated human platelets has been evaluated using an agarose gel technique. Supernatants of opsonized zymosan-stimulated platelets showed significant chemotactic activity (migration index $=$ $300 \pm 50$ ), in comparison with supernatants prepared from platelet suspensions stimulated with nonopsonized-zymosan (migration index $=10 \pm 15$ ) or resting platelet supernatants (migration index $=15 \pm 15$ ). Furthermore, a marked increase in chemotactic activity of the opsonized zymosan-treated platelet supernatants was demonstrated after the additon of NADH (migration index $=525 \pm 100$ ). The inclusion of specific inhibitors of the cycloxygenase and lipoxygenase pathways resulted in a marked reduction of chemotactic activity, which was restored in the presence of NADH. Further, the addition of superoxide dismutase completely abolished the chemotactic response induced by NADH. These data suggest that platelets are the source of chemotactic factor(s) derived from the activation of a superoxide generating system.
\end{abstract}

\section{Speculation}

Platelets play an important role in the nonspecific immune mechanisms by participating in the recruitment of inflammatory cells to the site of inflammation and in the cell-to-cell interaction. This function appears to be related to the production of chemotactic factors some of which are associated with a superoxide generating system.

Previous investigations have demonstrated that human platelet suspensions generate superoxide $\left(\mathrm{O}_{2}{ }^{-}\right)$anions at rest and after aggregation (17). It is now evident that the production of oxidizing substances by platelet suspensions is not due to polymorphonuclear leukocyte (PMN) contamination (16) and/or to passive diffusion from other cells (14). Using a cytochemical, ultrastructural method, we have recently demonstrated that platelets possess an $\mathrm{H}_{2} \mathrm{O}_{2}$ producing, $\mathrm{NADH}$-dependent system that is stimulated by interactions with particulate material (8).

Considerable accumulated evidence indicates that $\mathrm{H}_{2} \mathrm{O}_{2}$, and perhaps superoxide anions, released by granulocytes play a critical role in oxygen-dependent microbicidal activity (22). Platelets, although phagocytic, do not kill ingested bacteria (5).

To date, the physiologic role of the platelet oxidizing substance(s) generating system is not completely understood. Present evidence suggests that this system could be involved in the production of chemotactic factors. Perez et al. (20) suggested that an $\mathrm{O}_{2}{ }^{-}$generating system can produce chemotactic lipids from arachidonic acid. Moreover, it has been proposed that superoxide radicals play a role in cell-to-cell interaction between inflammatory cells $(10,18)$. In this way, generation of superoxide could provide a self-amplifying communicatioin system by reacting with an inactive plasma component, converting it to an active, stable and potent chemotactic factor (21).

Because platelets are involved in the inflammatory response and in immunologic processes $(2,4)$, the present study was performed to investigate whether PMN movement could be influenced by the platelet superoxide generating system.

Previous investigations have demonstrated the role of opsonized zymosan in the stimulation of human platelets $(19,31)$ and in the activation of the NADH-dependent, cyanide-insensitive, oxidase system (9). We now report that supernatants of opsonized zymosan-treated platelets, after inhibition of prostaglandin pathways, exhibit chemotactic activity for PMN and that this activity can be completely abolished by superoxide dismutase (SOD).

\section{MATERIALS AND METHODS}

Preparation of platelet suspension. Purified suspension of platelets were prepared from fresh blood samples using $2 \%$ ethylene diaminetetracetic acid (EDTA, BDH) $(9: 1 \mathrm{v} / \mathrm{v})$ as anticoagulant. Only those volunteers free of any drug treatment for at least 10 days were chosen. Particular care was taken to minimize leukocyte contamination (less than 1 leukocyte per $10^{8}$ platelets). A plateletrich preparation (PRP) was obtained by centrifugation of blood samples at $100 \times g$ for $15 \mathrm{~min}$ at room temperature. The supernatants were then gently layered on Ficoll (Ficoll 400, Pharmacia) and centrifuged at $80 \times g$ for $20 \mathrm{~min}$. The resulting supernatants were mixed with an equal volume of Dulbecco's phosphate buffer saline (PBS, Gibco) and washed twice.

Activation of platelet suspension. Zymosan A (Sigma) was boiled in distilled water for $20 \mathrm{~min}$, washed three times in $0.15 \mathrm{M} \mathrm{NaCl}$, incubated $(50 \mathrm{mg} / \mathrm{ml})$ with autologous, citrated plasma for $30 \mathrm{~min}$ at $37^{\circ} \mathrm{C}$ and then washed three more times in Dulbecco's PBS. The PRPs were then activated by mixing with an equal volume of opsonized zymosan for $30 \mathrm{~min}$ at $37^{\circ} \mathrm{C}$. The generation of prostaglandin products derived from the cycloxygenase pathway was inhibited by the addition of $100 \mathrm{mM}$ aspirin (ASA, Bayer). Both the cycloxygenase and lipoxygenase pathway were inhibited by the addition of $300 \mu \mathrm{g} / \mathrm{ml}$ of 5,8,11,14-eicosatetraenoic acid (ETYA, RO 3-1428, Roche). One mM KCN (Merck) was used to inhibit mitochondrial respiration and $10 \mu \mathrm{g} / \mathrm{ml}$ theophylline (Sigma) were added to inhibit platelet aggregation and granule protein release. The zymosan-activated platelet suspensions were then evaluated for the generation of $\mathrm{O}_{2}{ }^{-}$anions and $\mathrm{O}_{2}{ }^{-}$-mediated 
generation of chemotactic factors unrelated to prostaglandin metabolism by the addition of $0.5 \mathrm{mM}$ NADH (Sigma). To inhibit the NADH-dependent oxidase system, $50 \mu \mathrm{g} / \mathrm{ml}$ SOD (Sigma) was used. Heat-inactivated SOD was also employed as the control experiment. Control experiments were carried out using supernatants from: (1) resting platelets; (2) resting platelets plus inhibitors; (3) resting platelets plus inhibitors and NADH; (4) nonopsonized zymosan-activated platelets; (5) nonopsonized zymosan-activated platelets plus inhibitors; and (6) opsonized zymosan alone without platelets (tables 1 and 2). After incubation, the platelet suspensioins were centrifuged at $200 \times g$ for $20 \mathrm{~min}$, and the cell-free supernatants collected and used as sources of chemotactic factor(s) for PMNs.

Evaluation of chemotactic activity. Each supernatant was dialyzed against polyethylene glycol (PEG 6000, BDH), in order to concentrate the active component(s), filtered through Millipore filters $(0.45 \mathrm{micron})$, and then evaluated for chemotactic activity using normal human PMNs as responding cells in an agarose assay system (6). Briefly, $1.5 \mathrm{ml}$ of a $1.2 \%$ agarose (Reo 15 , Boehring) solution in RPMI 1640 (Gibco) with $10 \%$ fetal calf serum (FCS, Flow) were added to $35 \times 10 \mathrm{~mm}$ tissue culture dishes. Three wells ( $4 \mathrm{~mm}$ in diameter and spaced $2 \mathrm{~mm}$ apart) were cut using a suitable template punch. Suspensions of PMNs were obtained from blood samples of healthy volunteers according to methods previously described (7), and used at a concentration of $30 \times 10^{6} \mathrm{cells} / \mathrm{ml}$. Each test was performed in duplicate; $10 \mu 1$ of the cell suspension were added to the central well and $10 \mu \mathrm{l}$ of each supernatant or zymosan-activated serum, as positive control, were added to one of the lateral wells. The other well was filled with $10 \mu \mathrm{l}$ of RPMI 1640, as negative control. The dishes were

Table 1. Chemotactic activity of stimulated and unstimulated platelet supernatants

\begin{tabular}{lcc}
\hline \multicolumn{1}{c}{ Supernatants } & $\begin{array}{c}\text { M.I. } \\
\text { (mean } \pm \text { S.D.) }\end{array}$ & $\begin{array}{c}\text { Number of } \\
\text { experiments }\end{array}$ \\
\hline Resting platelets & $15 \pm 15$ & 4 \\
$\begin{array}{l}\text { Opsonized zymosan } \\
\text { Nonopsonized zymosan-stimulated }\end{array}$ & $12 \pm 10$ & 4 \\
$\quad \begin{array}{l}\text { platelets } \\
\text { Opsonized zymosan-stimulated plate- }\end{array}$ & $300 \pm 50$ & 4 \\
$\quad$ lets & & 4 \\
Resting platelets + NADH & $20 \pm 15$ & 4 \\
$\begin{array}{l}\text { Opsonized zymosan-stimulated platelets } \\
\text { + NADH }\end{array}$ & $525 \pm 100$ & 4 \\
\hline
\end{tabular}

${ }^{1} P<0.001$ when compared with resting platelets, or nonopsonized zymosan-stimulated platelets.

${ }^{2} P<0.01$ when compared with opsonized zymosan-stimulated platelets.

Table 2. Chemotactic activity of stimulated and unstimulated platelet supernatants + inhibitors 1

\begin{tabular}{|c|c|c|}
\hline Supernatants & $\begin{array}{c}\text { M.I. } \\
\text { (mean } \pm \text { S.D.) }\end{array}$ & $\begin{array}{l}\text { Number of } \\
\text { experiments }\end{array}$ \\
\hline Resting platelets \pm inhibitors & $18 \pm 10$ & 4 \\
\hline Resting platelets + inhibitors + NADH & $12 \pm 20$ & 4 \\
\hline $\begin{array}{l}\text { Nonopsonized zymosan-stimulated plate- } \\
\text { lets }+ \text { inhibitors }\end{array}$ & $10 \pm 5$ & 4 \\
\hline $\begin{array}{l}\text { Opsonized zymosan-stimulated platelets } \\
\quad+\text { inhibitors }\end{array}$ & $10 \pm 8$ & 5 \\
\hline $\begin{array}{l}\text { Opsonized zymosan-stimulated platelets } \\
+ \text { inhibitors }+\mathrm{NADH}^{2}\end{array}$ & $320 \pm 100$ & 5 \\
\hline $\begin{array}{l}\text { Opsonized zymosan-stimulated platelets } \\
+ \text { inhibitors + NADH + SOD }\end{array}$ & $25 \pm 20$ & 3 \\
\hline $\begin{array}{l}\text { Opsonized zymosan-stimulated platelets } \\
+ \text { inhibitors + NADH + heat-unacti- } \\
\text { vated SOD }\end{array}$ & $300 \pm 100$ & 3 \\
\hline
\end{tabular}

covered and incubated in a $37^{\circ} \mathrm{C}$ humidified atmosphere with $5 \%$ $\mathrm{CO}_{2}$ for $3 \mathrm{~h}$. Afterwards, the preparations were fixed with MayGrunwald (Hoechst) for $30 \mathrm{~min}$, the agarose layers removed, and the plates stained with Giemsa (Hoechst). The migrated cells were counted, using a $10 \times$ eyepiece grid $(10 \times 10 \mathrm{~mm})$ at $\mathrm{X} 10$ magnification. The number of cells randomly migrating towards the buffer was subtracted from the number of cells migrating directly toward the attractant to obtain the net chemotactic response. Each preparation was also projected using a Leitz microprojector (magnification scale $1: 175$ ) and the maximum distance of migration was measured and the distance of random migration was subtracted. The migration index (M.I.) was then calculated by the following formula:

$$
\text { M.I. }=\frac{\left(N_{c a}-N_{b}\right) \times\left(D_{c a}-D_{b}\right)}{100}
$$

Where $N_{\text {ca }}$, number of cells which had migrated toward the chemoattractant (ca); $\mathrm{N}_{\mathrm{b}}$, number of cells which had migrated toward the buffer (b); $D_{c a}$, the maximum migration distance toward the chemoattractant (ca); and $\mathrm{D}_{\mathrm{b}}$, the maximum migration distance toward the buffer.

Platelet studies. In order to verify the effect of various inhibitors on platelet metabolism and function, $\mathrm{O}_{2}$ consumption, before and after the addition of the inhibitors, was measured polarographicaly with a Clark's electrode (Yellow Spring Instruments Co., Yellow Spring, $\mathrm{OH}$ ), in the presence or in the absence of thrombin (final concentration, $1.7 \mathrm{U} / \mathrm{ml}$ ).

Platelet aggregation was measured photometrically (3), using an aggregometer (ELVI).

\section{RESULTS}

The results of chemotactic activity of supernatants derived from stimulated and unstimulated platelets after various treatments are shown in Table 1.

The M.I. obtained testing normal PMNs with opsonized zymosan was $522 \pm 222$. This has been considered as positive control.

The supernatants of opsonized zymosan-stimulated platelets showed significant chemotactic activity (M.I. $=300 \pm 50, P<$ 0.01 ; Student $t$ test), in comparison with supernatants prepared from platelet suspensions stimulated with nonopsonized-zymosan (M.I. $=10 \pm 15$ ), or resting platelet supernatants (M.I. $=15 \pm$ 15). The addition of NADH resulted in a marked increase of chemotactic activity in the suspensioins of opsonized zymosantreated platelets (M.I. $=525 \pm 100, P<0.01$ ).

The results of studies designed to determine the effect of inhibitors on the chemotactic activity are shownin Table 2 . It can be seen that the inclusion of specific inhibitors of the cycloxygenase and lipoxygenase pathways, i.e., ASA and ETYA, significantly reduced the chemotactic activity of supernatants derived from opsonized zymosan-stimulated platelets $($ M.I. $=10 \pm 8$ ); nevertheless, the stimulated platelets still retained the capacity to generate chemotactic factor(s) in the presence of NADH (M.I. $=300$ $\pm 100, P<0.001)$. This chemotactic activity was completely abolished by the addition of SOD (M.I. $=25 \pm 20$ ), whereas unactivated SOD did not modify the chemotactic activity (Table 2).

The platelet function was completely inhibited, as demonstrated by the aggregation studies; moreover, the inhibition of prostaglandin pathways by ASA and ETYA was verified by the lack of thrombin-induced oxygen consumption in the treated platelet suspensions. In control experiments, the supernatants of PMN suspensions, as well as mononuclear cells, at concentrations equivalent to any possible contamination of PRP failed to generate chemotactic activity, either in the presence or absence of NADH.

\section{DISCUSSION}

The results of the present investigations suggest that significant chemotactic activity is produced by platelets in the presence of 
NADH after challenge with osponized zymosan. Superoxide radicals were required as shown by essentially complete inhibition by SOD.

Previous studies have demonstrated that after stimulation of PMNs and monocytes by any of a variety of stimuli (e.g., particulate challenge), a membrane perturbation that is associated with $\mathrm{NAD}(\mathrm{P}) \mathrm{H}$ oxidase activation, occurs, which leads to the formation of superoxide radicals (1). Our data, showing an increase of PMN chemotactic response to stimulated platelet supernatants after the addition of NADH and its inhibition by SOD, strongly suggest that production and/or release of factor(s) with chemotactic activity for PMNs could be linked to a superoxide-generating system. The possibility that superoxide anions could be produced by contaminating PMNs can be excluded by our control experiments; moreover, previous studies from our laboratory have suggested that in purified platelet preparations, the contribution of PMNs in the production of superoxide radicals appears to be negligible (8).

Chemotactic factors are heterogenous in type and appear to arise from widely different sources $(2,27)$. Under the present experimental conditions, in which the presence of exogenous factors such as bacterial products can be excluded, both cellular and humoral factors appear to be responsible for PMN chemotaxis. In the present studies, which were conducted in absence of plasma and serum, the possibility that serum derived chemotactic factors as well as those derived from coagulation and fibrinolytic systems, or from kinin-generating pathways, could also be excluded. In addition, chemotactic activity was not demonstrated with mononuclear cell contamination (up to 1 cell $/ 10^{3}$ platelets). The present study, therefore, suggests that platelets themselves generate chemotactic activity. To date, two different mechanisms of generations of chemotactic activity by platelets have been reported. Stimulation of platelets can induce release of a chemotactic factor from granules which interacts with the complement cascade (29); moreover, it has been suggested that oxygenate arachidonate products via lipoxygenase, are potent chemotactic factors $(13,15,26)$. The first hypothesis could be ruled out because the experiments were carried out in the absence of serum and in the presence of platelet aggregation and release inhibitors (cyanide, theophylline, ....). Perturbation of platelet membranes leads to mobilization of arachidonic acid from membrane phospholipids (24); the mobilized arachidonic acid may subsequently be oxidized by either cycloxygenase and/or lipoxygenase pathways. Products of both cycloxygenase, i.e., 12-L-hydroxy-5,8,10-heptadecatrienoic acid, HHT, and lipoxygenase pathways, i.e., 12-L-hydroxy-5,8,10,14-eicosatetraenoic acid, HETE, are potent chemotactic factors for human eosinophils and neutrophils $(15,28)$. Anti-inflammatory agents such as aspirin and indomethacin inhibit the cycloxygenase pathway whereas the acetylenic compound 8,11,14-eicosatetraenoic acid (ETYA) blocks both pathways $(11,23,30)$. In the present study, chemotactic activity for PMNs was still present (in the presence of NADH) when lipoxygenase and cycloxygenase inhibitors were added to the platelet suspensions. This finding excludes the possibility that the platelet-derived chemotactic factor(s) arise from arachidonate metabolites and suggests that a superoxide-generating system is involved. It has been shown that nonenzymatic oxidation of arachidonic acid may generate biologic chemotactic factor without the activation of either lipoxygenase or complement system (25). Fridovich and Porter (12) demonstrated that hydroperoxides are formed by superoxide-mediated arachidonic acid oxidation. Their experiments provide a direct chemical link with findings reported by other investigators. Petrone et al. (21) found that superoxide can react with a precursor in plasma to generate a factor that is chemotactic for neutrophils. This generation was strictly superoxide-dependent because it was inhibited by superoxide dismutase, but not catalase; moreover, it has been reported that the in vitro incubation of purified arachidonic acid with a superoxide-generating system results in the formation of chemotactic products (20).

The present study demonstrates that platelets themselves are also capable of generating chemotactic activity in the absence of plasma and in the presence of NADH. Because NADH does not cross the membrane, our data support the hypothesis that a $\mathrm{NADH}$-dependent-oxidizing substance-generating system is present on platelet plasma membrane. Finally, it has been shown that platelet stimulation by a number of agents is associated with membrane alterations that lead to increased enzymne activities. One possible explanation of the present results is that perturbation of membranes by opsonized-zymosan activates an oxidase system, leading to the generation of superoxide radicals. These $\mathrm{O}_{2}{ }^{-}$radicals can, in turn, react with a platelet component resulting in the production of factor(s) highly chemotactic for PMNs.

In conclusion, although further investigations are necessary to better clarify the specific nature of this factor(s) and its chemical characteristics, the present studies suggest that platelets play an important role in the nonspecific immune response by participating in the recruitment of inflammatory cells to the site of inflammation. This platelet function appears to be related to the production of chemotactic factors, some of which are associated with a superoxide-generating system.

\section{REFERENCES AND NOTES}

1. Babior, B. M.: Oxygen-dependent microbial killing by phagocytosis. N. Engl. J Med., 298: 659 (1978).

2. Becker, E. L.: Chemotaxis. J. Allergy Clin. Immunol., 66: 97 (1980).

3. Born, G. V. R.: Aggregation of blood platelets by adenosine diphosphate and its reversal. Nature, 194: 927 (1962)

4. Brown, D. L.: Platelets in immunological reactions. In: Gorman, J. L., Ed.: Platelets in Biology and Pathology. (Elsevier North Holland Biomedical Press, 1976).

5. Clawson, C. C. and White, J. C.: Platelet interaction with bacteria. II. Fate of the bacteria. Am. J. Pathol., 65: 381 (1971).

6. D'Amelio, R., Rossi, P., Le Moli, S., and Aiuti, F.: Neutrophil chemotaxis defect in IgA deficiency evaluated by migration agarose method. Scand. J. Immunol., 11: 471 (1980)

7. D'Amelio, R., Rossi, P., Le Moli, S., and Aiuti, F.: Defective neutrophil chemotaxis in hypogammaglobulinemia and selective IgA deficiency. Clin. Immunol Immunopath., 16: 287 (1980).

8. Del Principe, D., Mancuso, G., Menichelli, A., Gabriotti, M., Cosmi, E. V., and Gherardi, G.: Production of hydrogen peroxide in phagocytizing human platelets: an electron microscopic cytochemical demonstration. Biol. Cell, 38 : 135 (1980).

9. Del Principe,D., Mancuso, G., Menichelli, A., Gabriotti, M., and D'Arcangelo, C.: NADH-dependent $\mathrm{H}_{2} \mathrm{O}_{2}$ production in human blood platelets. In: Proceedings of the EMBO Workshop on platelets: cellular response, mechanisms and their biological significance. Rehovot, April 13-19 (1980).

10. Dormandy, T. L.: Free-radicals oxidation and antioxidants. Lancet, $1: 647$ (1978).

11. Flower, R. J.: Drugs which inhibit prostaglandins biosynthesis. Pharmacol. Rev., 26: 33 (1974).

12. Fridivich, S. E. and Porter, N. A.: Oxidation of arachidonic acid in micelles by superoxide and hydrogen peroxide. J. Biol. Chem., 256: 260 (1981).

13. Goetzl, E. J., Woods, J. M., and Gorman, R. R.: Stimulation of human eosinophil and neutrophil polymorphonuclear leukocyte chemotaxis and random migration by 12-L-hydroxy-5,8,10,14-eicosatetraenoic acid. J. Clin. Invest., 59: 179 (1977).

14. Handin, R. 1., Karabin, R., and Boxer, G. J.: Enhancement of platelet function by superoxide anions. J. Clin. Invest., 59: 959 (1977).

15. Higgs, G. A., Moncada, S., and Vane, J. R.: The role of arachidonic acid metabolism in inflammation. In: Gerald Weissmann, Ed., Bengt Samuelsson. Ed., Rodolfo Paoletti, Ed.: Advances in Inflammatioin Research, Vol. I., pp. 413-430 (Raven Press, New York, 1979).

16. Krinsky, R. I., Scoon, K. S., Hardin, J. C., and Levine, P. H.: Source of hydrogenperoxide and chemiluminescence observed in activated human platelet preparations. Blood, 50: 597 (1978).

17. Marcus, A. J., Silk, S. T., Safier, L. B., and Lillman, H. L.: Superoxide production and reducing activity in human platelets. J. Clin. Invest., 59: 149 (1977).

18. McCord, J. M., Stokes, S. H., and Wong, K.: Superoxide radicals as a phagocyteproduced chemical mediator of inflammation. In: Gerald Weissmann, Ed., Bengt Samuelsson, Ed., Rodolfo Paoletti, Ed.: Advances in Inflammation Research, Vol. I, pp. 273-280 (Raven Press, New York, 1979).

19. Pfueller, S. L. and Luscher, E. F.: Studies on the mechanism of the human platelets release from reaction induced by immunologic stimuli. II. The effect of zymosan. J. Immunol., 112: 1211 (1974).

20. Perez, M. D., Weskler, B. B., and Goldstein, I. M.: Generation of chemotactic lipids from arachidonic acid by exposure to a superoxide generating system. Inflammation, 4: 313 (1980).

21. Petrone, W. F., English, D. K., Wong, K., and McCord, J. M.: Free radicals and inflammation. Superoxide dependent activation of a neutrophil chemotactic factor in plasma. Proc. Natl. Acad. Sci. USA, 77: 1159 (1980).

22. Root, R. K., Metcalf, J., Oshino, M., and Chang, E. G.: $\mathrm{H}_{2} \mathrm{O}_{2}$ release from human granulocytes during phagocytosis. I. Documentation, quantitation and some regulatory factors. J. Clin. Invest., 55: 945 (1975). 
23. Roth, G. I. and Majereus, P. W.: The mechanism of the effect of aspirin on human platelets. J. Clin. Invest., 56: 624 (l975).

24. Samuelsson, B., Gldyne, M., Ganstrom, E., Hamberg, M., Hammarstrom, S., and Malmsten, C.: Prostaglandins and thromboxanes. Ann. Rev. Biochem., 47: 997 (1978).

25. Turner, S. R., Campbell, J. A., and Lynn, W. S.: Polymorphonuclear leukocyte chemotaxis toward oxidized lipid components of cell membrane. J. Exp. Med., 141: 1437 (1975).

26. Turner, S. R., Tainer, J. A., and Lynn, W. S.: Biogenesis of chemotactic molecules by the arachidonate lipoxygenase system of platelets. Nature, 257: 680 (1975).

27. Valone, F. M.: Modulation of human neutrophil and eosinophil polymorphonuclear leukocytes chemotaxis: an analytical revue. Clin. Immunol. Immunopath., 15:52(1980).

28. Weissmann, G., Smollen, J. E., and Korchak, H. M.: Release of inflammatory mediators from stimulated neutrophils. N. Engl. J. Med., 303: 27 (1980).
29. Weskler, B. B. and Coupal, C. E.: Platelet dependent generation of chemotactic activity in serum. J. Exp. Med., 137: 1419 (1973)

30. Willis, A. L., Kuhn, D. C., and Weiss, H. I.: Acetylenic analog of arachidonates that acts like aspirin on platelets. Science, 183: 327 (1974)

31. Zucker, B. M. and Grant, R. A.: Aggregation and release reaction induced in human blood platelets by zymosan. J. Immunol., 112: 1219 (1974).

32. The authors are indebted to Dr. Giovanna Mancuso for her technical assistance and to Dr. Lata Nerurkar (Georgetown University, School of Medicine, Washington D.C.) for kindly reviewing the manuscript.

33. Requests of reprints should be addressed to: Dr. Paolo Rossi, Section of Allergy and Clinical Immunology, $\mathrm{I}^{\circ}$ Department of Pediatrics, University of Rome, Viale Regina Elena, 324, 00161, Rome, Italy.

34. Received for publication June 24, 1981

35. Accepted for publication February 23, 1982. 